\title{
EVIDÊNCIAS SOBRE MUDANÇA ESTRUTURAL, HETEROGENEIDADE ESTRUTURAL E REGIONAL: UMA ANÁLISE DA PRODUTIVIDADE DO TRABALHO BRASILEIRA NO PERÍODO 2004-2015
}

Thais Andreia Araujo de Souza ${ }^{1}$ Marieli Vieira ${ }^{2}$ Marina Silva da Cunha ${ }^{3}$

Resumo: Este estudo objetivou estudar a produtividade do trabalho brasileira (20042015) buscando quais unidades federativas e setores mais contribuíram para o baixo desempenho. Concluiu-se que há crescimento da produtividade, sendo verificado que a agropecuária possuiu menor nível e maior crescimento, e a região Nordeste possuiu menor nível também e maior crescimento. Também foi constatado que o baixo desempenho da produtividade decorreu principalmente da produtividade intrassetorial. Portanto, residiu na falta de crescimento dentro dos setores, e, a mudança estrutural contribuiu pouco para o desempenho, portanto, uma aceleração desse processo de mudança na estrutura produtiva beneficiaria a produtividade total. Ademais, conclui-se que existe heterogeneidade estrutural e regional no país.

Palavras-chave: Heterogeneidade estrutural. Heterogeneidade regional. Mudança estrutural. Produtividade intrassetorial.

\section{EVIDENCE ON STRUCTURAL CHANGE, STRUCTURAL AND REGIONAL HETEROGENEITY: AN ANALYSIS OF THE PRODUCTIVITY OF BRAZILIAN LABOUR IN THE PERIOD 2004-2015}

\begin{abstract}
This article aimed to study the Brazilian labour productivity (2004-2015) searching for which federative units and sectors contributed most to its low performance. It was concluded that there is productivity growth, being verified that the agriculture had lower level and higher growth, and the Northeast region had lower level and higher growth. It was also found that poor productivity performance was mainly due to intrasectoral productivity. Therefore, it resided in the lack of growth within the sectors, and the structural change contributed little to its performance, so an acceleration of this processo of change in the productive structure would benefit the total productivity. In addition, it is concluded that there is structural and regional heterogeneity in the country.
\end{abstract}

Keywords: Structural heterogeneity. Regional heterogeneity. Structural change. Intrasectorial productivity.

\section{EVIDENCIA SOBRE EL CAMBIO ESTRUCTURAL, LA HETEROGENEIDAD ESTRUCTURAL Y REGIONAL: UN ANÁLISIS DE LA PRODUCTIVIDAD LABORAL BRASILEÑA EN EL PERÍODO 2004-2015}

Resumen: Este estudio tuvo como objetivo estudiar la productividad laboral brasileña (2004-2015), buscando qué sectores y unidades federativas contribuyeron

\footnotetext{
1 Universidade Federal do Rio Grande do Sul (UFRGS), Departamento de Economia e Relações Internacionais, Porto Alegre, Brasil, thaisandreiaa@gmail.com, https://orcid.org/0000-0001-9605-4895 2 Universidade Federal do Rio Grande do Sul (UFRGS), Departamento de Economia e Relações Internacionais, Porto Alegre, Brasil, marihvieira18@gmail.com, https://orcid.org/0000-0002-0446-3235 3 Universidade Estadual de Maringá (UEM), Departamento de Economia, Maringá, Brasil, mscunha@uem.br, https://orcid.org/0000-0001-9122-3944
} 
más al bajo desempeño. Se concluyó que hay un crecimiento en la productividad, se verificó que la agricultura tuvo un nível más bajo y un mayor crecimiento, y que la región del Nordeste tuvo un nível más bajo y un mayor crecimiento. También se descubrió que el bajo desempeño de la productividad se debía principalmente a la productividad intrasectorial. Por lo tanto, residía em la falta de crecimiento dentro de los sectores, y el cambio estructural contribuyó poco al desempeño, por lo que una aceleración de este processo de cambio en la estructura de producción beneficiaría la productividad general. Además, se concluye que existe una heterogeneidade estructural y regional en el país.

Palabras clave: Heterogeneidad estructural. Heterogeneidad regional. Cambio estructural. Productividad intrasectorial.

\section{Introdução}

Frente ao cenário de crescimento econômico, a produtividade tem sido um tema importante para o Brasil. Isso porque, segundo De Negri e Cavalcante (2014), a economia brasileira não foi influenciada a longo prazo pelos investimentos, e, a indústria, considerada por Kaldor (1978), o motor da economia, perdeu lugar na composição do PIB para o setor de serviços, portanto, há espaço para que a economia cresça com base na produtividade. Mas, de acordo com Squeff e De Negri (2014), a eficiência produtiva do Brasil já vinha apresentando baixo desempenho desde a década de 1980, com pequeno crescimento apenas nos anos 2000, desse modo, é importante verificar como se apresenta o desempenho recente da produtividade.

Alguns trabalhos se destacam na literatura empírica nacional quando se trata do estudo da produtividade do trabalho para o Brasil, considerando a importância da mão de obra para o crescimento econômico (Bonelli e Veloso, 2012; Bonelli e Bacha, 2013; Cavalcante e De Negri, 2014). Os trabalhos de Matteo (2015) e Nogueira, Infante e Mussi (2014), por exemplo, analisam as diferenças regionais da produtividade do trabalho. Também a análise setorial se constitui em uma área de interesse (Bonelli, 2014; Galeano e Wanderley, 2013; Fochezatto e Stulp, 2007; Veloso, Matos e Coelho, 2015). Entre as conclusões encontradas por estes estudos, se verificou que o que caracteriza o comportamento da produtividade brasileira é o maior crescimento alcançado por setores e regiões pouco dinâmicas. Ou seja, os setores e regiões que mais tinham poder para influenciar positivamente a produtividade agregada não tiveram bons desempenhos.

De acordo com o exposto, este trabalho busca verificar como se dá o desempenho da produtividade do Brasil recente, evidenciando se este se caracteriza 
pela heterogeneidade regional ou estrutural e analisando também os efeitos de mudanças da estrutura produtiva sobre a estrutura agregada, considerando o período entre 2004 e 2015. Para este objetivo, a produtividade do trabalho é calculada com base no total de pessoal ocupado em cada atividade econômica, sendo definida como a razão entre o PIB e pessoal ocupado. A contribuição deste trabalho se dá pela análise das metodologias de maneira desagregada, com a produtividade por macrossetor (agropecuária, indústria e serviços) e por unidades federativas. Busca-se saber quais regiões e setores mais contribuíram para o baixo desempenho agregado.

A heterogeneidade estrutural corresponde à considerável disparidade, que se perpetua no tempo, entre os diferentes níveis de produtividade dos agentes econômicos, conforme Squeff e Nogueira (2013). Enquanto a heterogeneidade regional, derivando desta última, é um diferencial que pode ocorrer também entre as regiões de um país, não só com disparidades na produtividade entre as regiões, mas também entre os setores de atividades dentro destas regiões, de acordo com Matteo (2015). Quanto à mudança estrutural, Squeff e De Negri (2014) a explicam como as modificações nas estruturas produtivas dos países devido à migração dos trabalhadores entre setores menos e mais produtivos.

Buscando descobrir se a migração de mão de obra entre os setores agropecuária, indústria e serviços impactou no crescimento da produtividade do trabalho, assim como a produtividade do trabalho entre os setores, foi realizada uma decomposição desse crescimento entre efeito direto (produtividade intrassetorial), efeito ocupação (mudança estrutural), efeito preços e efeito interação.

A estrutura deste trabalho se encontra dividida em cinco seções além desta introdução. Na sequência foi realizada uma discussão teórica acerca da relação produtividade do trabalho e crescimento econômico. Na terceira seção encontram-se evidenciadas algumas análises a respeito do comportamento da produtividade brasileira, com destaque para as mais desagregadas. A quarta seção apresenta a metodologia utilizada para estimar a produtividade do trabalho e a decomposição desta. Por último, é feita a exposição e discussão das estimativas e da composição de produtividade do trabalho, finalizando com as considerações finais. 


\section{Revisão teórica}

De acordo com a segunda lei de Kaldor, ou lei de Kaldor-Verdoorn, a produtividade do trabalho pode ser relacionada com o crescimento econômico, e quanto mais rápida fosse a taxa de crescimento da indústria manufatureira mais cresceria a produtividade no setor, sendo justificada pelas economias de escala e retornos crescentes (Thirwall, 1983). Os retornos à escala se relacionavam à interação entre a elasticidade da oferta e demanda dos bens manufatureiros, e esta interação seria causadora do efeito positivo entre produtividade e produto na manufatura.

Kaldor (1978) investigou o porquê de a taxa de crescimento do Reino Unido ser baixa na primeira metade do século $X X$, e verificou que a taxa de crescimento econômico se associaria à taxa de crescimento de manufatura em uma fase intermediária de desenvolvimento econômico, na qual a economia se moveria de imatura para madura. Para o autor, a taxa de crescimento do Reino Unido era baixa porque a economia chegou à maturidade antes dos outros países e seu potencial para o rápido crescimento foi exaurido antes que se atingissem altos níveis de produtividade. Deste modo, o Reino Unido sofreu de maturidade prematura.

Outrossim, quanto maior a elevação do produto da indústria dentro do setor de manufatura, maior seria a transferência de mão de obra de setores que apresentassem retornos decrescentes, fazendo com que a produtividade desses setores crescesse. Entende-se, portanto, que quanto maior a taxa de crescimento da produtividade dentro do setor da manufatura, maior o impacto na produtividade da economia como um todo, implicação que se conhece como terceira lei de Kaldor, de acordo com Thirwall (1983). O aumento da produtividade geral provocado pela manufatura tenderia a diminuir, assim como a taxa de crescimento do produto, quando a transição de mão de obra de outros setores aumentasse ou quando a produção passasse a depender de todos os setores da economia.

O processo de mudança estrutural seria traduzido pelo acontecimento de que os países mais desenvolvidos teriam essa mão de obra deslocada do setor de agricultura para o setor de indústria e de serviços, ocasionando a queda no crescimento da produção. Isto é, quanto mais desenvolvidos os países, maior seria a substituição de mão de obra por capital físico na agropecuária, mão de obra essa que seria absorvida pela indústria ocasionando aumento da produtividade nos dois setores. A fase seguinte seria aquela em que a mão de obra passaria a ser absorvida pelo setor de serviços, caracterizando uma nova mudança estrutural 
(Baumol, 1967). Desse modo, se verifica que a produtividade tem um papel muito correlacionado ao do crescimento dos setores e da economia agregada.

\section{Evidências empíricas na literatura para o caso brasileiro}

Nesta seção veremos alguns trabalhos acerca da produtividade do trabalho no Brasil que têm destaque na literatura empírica. Iniciamos pelo trabalho de Bonelli e Veloso (2012), que buscaram verificar a taxa de crescimento médio da produtividade do trabalho, que foi de 1,2\% a.a. entre os anos de 1995 e 2009. No trabalho de Bonelli e Bacha (2013), os valores são menores, ficando em 0,36\% a.a. entre os anos de 1993 e 1999, e 0,67\% a.a. entre os anos de 2000 e 2009. Cavalcante e De Negri (2014), que investigaram os períodos de 1992 a 2001 e de 2001 a 2009, estimando as taxas médias de crescimento para esses períodos, encontraram os valores médios de crescimento de 1,09\% a.a. e 1,17\% a.a., respectivamente. Sendo que essas taxas são mais próximas às encontradas por Bonelli e Veloso (2012). Bonelli (2014) verificou que a produtividade do trabalho no setor de serviços foi maior que a produtividade total da economia, sendo que o crescimento foi mais lento que o do setor agropecuário.

O estudo de Matteo (2015), buscou analisar a produtividade brasileira no âmbito regional. O autor fez comparação entre as regiões brasileiras no período de 1996 a 2008 e considerando os setores agropecuário, indústria, construção, comércio e serviços. Verificou-se que a região Centro-Oeste teve produtividade 6,5 vezes maior que a região Nordeste, o que pode ser justificado pelo fato de a primeira ter como característica a pecuária extensiva, enquanto a segunda se baseia na agricultura familiar, conforme Nogueira, Infante e Mussi (2014). O estudo também observou que no Nordeste a produtividade do trabalho na agropecuária foi próxima da metade da produtividade brasileira, e a produtividade do setor de serviços representou cerca de $1 / 3$ do Sudeste. Concluiu-se que existiu heterogeneidade regional na produtividade do trabalho dentro do país, o que foi causado principalmente pelo fato de algumas regiões disporem de mais acesso ao capital para produção que outras.

Estudando a relação entre a produtividade na indústria e a intensidade tecnológica de maneira regional, Galeano e Wanderley (2013) concluíram que os setores que apresentavam mais alta tecnologia apresentavam também maior produtividade, porém essa alta produtividade não compensava o restante dos setores, que não possuíam tanta tecnologia. Entre as regiões, da mesma forma, o 
decréscimo da produtividade no Sudeste não era compensado pelo aumento desta nas demais regiões, fazendo com que houvesse estagnação na produtividade da indústria. Neste estudo também foi feita a desagregação da região Sudeste, onde se verificou que somente o estado de São Paulo teve decréscimo na produtividade do trabalho. Concluiu-se, portanto, que no ano de 2007, a produtividade do trabalho industrial estava abaixo da média nacional nas regiões Sul, Centro-Oeste e Nordeste, e que a região Sudeste estava acima da média, mas decrescendo. A região que obteve a maior produtividade do trabalho foi a região Norte.

Fochezatto e Stulp (2007), estudaram a produtividade do trabalho de forma regional na década de 1990, onde verificaram que de oito setores, três teriam se encaminhado para uma produtividade mais alta, melhorando o desempenho nos estados da região Sul, Sudeste e Centro-Oeste. Segundo os autores, a agropecuária deslocou-se para uma produtividade mais baixa, mas com bom desempenho nas regiões Sul e Centro-Oeste, enquanto o setor de serviços foi o que se dirigiu para a mais alta produtividade.

Desagregando o Brasil em setores para os anos de 1995 a 2013, Veloso, Matos e Coelho (2015) analisaram a produtividade do trabalho formal e informal e concluíram que houve queda na produtividade da indústria $(-0,4 \%)$, enquanto a produtividade na agropecuária cresceu, $6,3 \%$. No setor de serviços, os resultados indicam que houve um pequeno crescimento da produtividade nesse período, $0,7 \%$. O crescimento na eficiência produtiva da economia ao todo foi de $1,3 \%$, resultado que se aproxima ao desempenho descrito por De Negri e Cavalcante (2014) para o país.

Squeff e Nogueira (2013) verificaram que, a partir de 1980, enquanto a agropecuária perdeu participação, o setor de serviços teve um crescimento considerável e a indústria teve comportamento ínfimo, crescendo sua maior parcela na década de 1980. Quanto à produtividade do trabalho entre 1950 e 2009, somente o setor agropecuário obteve crescimento superior ao da economia como um todo. Até a década de 1970 o crescimento da produtividade foi distribuído em todos os setores da economia, situação que mudou nas décadas de 1980 e 1990, quando somente a agropecuária apresentou resultado positivo. Na década de 2000, o pequeno crescimento existente correspondeu à produtividade da agropecuária.

Uma das possíveis causas da queda no crescimento da década de 1990 é a liberalização comercial e financeira, de acordo com o que explicam Squeff e Nogueira (2013). Essa queda na produtividade ocorreu até o ano de 2004, com 
retomada do crescimento, que foi interrompido pela crise no ano de 2008. Também, segundo os autores, a heterogeneidade estrutural brasileira pode ser descrita por forte rigidez. Eles afirmam que o fato dos setores menos expressivos terem apresentado um crescimento superior aos demais setores em conjunto não significa que esteja havendo convergência entre os diferentes tipos de produtividade. Entre os resultados, verificou-se a existência de alguma rigidez na produtividade do trabalho, convergindo para baixo, em que se manteve heterogeneidade estrutural.

O trabalho de Bonelli (2014) mostrou que ainda era possível atuar, fazendo com que a mudança estrutural tivesse efeitos positivos na produtividade e que a produtividade intrassetorial teve um efeito maior. Similarmente, Squeff e De Negri (2014), verificaram que o crescimento intrassetorial teve mais efeito sobre a produtividade que a mudança setorial. Com metodologias diferentes, o trabalho de Bonelli (2014) partiu da decomposição nos componentes estruturais e intrassetoriais, enquanto o trabalho de Squeff e De Negri (2014), usou adicionalmente, a decomposição entre os itens preço e interação, indicando que o setor de serviços foi o responsável pela mudança estrutural na economia com comportamento relevante da produtividade, visto que era onde se concentrava a baixa produtividade.

De acordo com o exposto até aqui, vimos que a produtividade nos setores é importante para o crescimento e desenvolvimento do país. Constatou-se também que nas últimas décadas a produtividade da agropecuária brasileira tem apresentado um bom desempenho, contrastando com os setores da indústria e serviços. Os autores destacados também dão ênfase na existência da heterogeneidade estrutural e regional brasileiras, assim como ao processo de mudança estrutural, que pode auxiliar no desempenho da produtividade, portanto, o levantamento teórico corrobora as hipóteses levantadas no presente estudo.

\section{Procedimentos metodológicos}

O objetivo deste trabalho é analisar os efeitos de mudanças na estrutura produtiva sobre a produtividade agregada, além de destacar se há existência de heterogeneidade estrutural e regional entre as unidades federativas, sendo observado o período de 2004 a 2015 devido à disponibilidade dos dados ${ }^{4}$. Para isto, foram utilizados os dados de valor adicionado bruto a preços correntes e a preços do ano anterior e índice de preços por atividade econômica para as unidades

\footnotetext{
${ }^{4}$ Somente a partir do ano de 2004 a zona rural da região Norte foi incluída na pesquisa, e, o ano de 2015 foi o último em que houve a publicação da PNAD anual.
} 
federativas, sendo os dados obtidos das Contas Regionais (IBGE, 2019). E para mensuração do total de ocupações por atividade econômica e unidade federativa, foram utilizadas as bases de microdados das Pesquisas por Amostra de Domicílios Anuais (PNAD, 2019). Em seguida as atividades foram somadas para compor os setores de agropecuária, indústria e serviços. A produtividade do trabalho foi calculada conforme sugerido por Squeff e De Negri (2014):

$$
\hat{X_{t}^{i}}=\frac{Y_{t}^{i}}{L_{t}^{i}}
$$

Em que $\hat{X}_{t}^{i}$ se refere à produtividade do trabalho na atividade econômica $i \mathrm{e}$ ano $t, Y_{t} t_{t}$ se refere ao valor adicionado bruto deflacionado para o ano de 2010 na atividade econômica $i$ e ano $t$, e, $L_{t}^{i}$ se refere ao pessoal ocupado na atividade econômica $i$ e ano $t$. Ademais, com o objetivo de verificar a existência de heterogeneidade estrutural e regional, foi realizada uma análise por meio da observação dos níveis de produtividade do trabalho nos anos 2004 e 2015 e a taxa de crescimento no período por meio das unidades federativas. Para isto, a produtividade e sua respectiva taxa de crescimento foram divididas em quatro quartis, conforme metodologia de Squeff e Nogueira (2013). O intuito foi de verificar se houve diferença no desempenho da produtividade entre os setores e unidades, e, se esta diferença permaneceu constante durante o período, constatando heterogeneidade.

Em seguida, foi efetuada uma decomposição do crescimento da produtividade seguindo Squeff e De Negri (2014), em que foi utilizada a metodologia de análise shift-share. Esta metodologia contribui ao destacar o papel dos preços relativos para a produtividade, além das contribuições da produtividade intrassetorial e a parcela de ocupações por setor (captada como mudança estrutural):

$$
X_{t}=\frac{\sum_{i} Q_{t}^{i}}{P_{t} L_{t}}=\frac{\sum\left(Y_{t}^{i} P_{t}^{i} L_{t}^{i} / L_{r}^{i}\right)}{P_{t} L_{t}}=\frac{\sum\left(P_{t}^{i} L_{t}^{i} X_{t}^{i}\right)}{P_{t} L_{t}}=\sum\left(p_{t}^{i} s_{t}^{i} X_{t}^{i}\right)
$$

Em que $Q_{t}^{i}$ se refere ao valor adicionado a preços correntes, $P_{t}$ é o índice de preços do valor adicionado agregado, $p_{t}^{i}=P_{t}^{i} / P_{t}$ se refere aos preços relativos e $s_{t}{ }_{t}=L_{t}{ }^{i} / L_{t}$ se refere à participação das ocupações na atividade econômica no total de ocupações. Na sequência, é definido $q_{t}^{i}=Q_{t}^{i} / Q_{t}$ como a participação da atividade econômica no valor adicionado agregado a preços correntes, $\hat{X}_{t}=\left(X_{t}-X_{t-1}\right) / X_{t-1}$ como a variação da produtividade agregada no período, $\hat{X}_{t}^{i}=\left(X_{t}^{i}-X_{t-1}^{i}\right) / X_{t-1}^{i}$ como 
a variação da produtividade de cada atividade econômica no período, $\sigma^{i}=\left(s_{t}^{i}-s_{t-1}^{i}\right) / s_{t-1}^{i}$ como a variação no share de ocupações no período, e, $\rho^{i}=\left(p_{t}^{i}-p_{t-1}^{i}\right) / p_{t-1}^{i}$ como a variação dos preços relativos no período. Finalmente, definidos os conceitos, é possível observar como se decompõe o crescimento da produtividade:

$$
\hat{X}_{t}=\sum_{i} q_{t-1}^{i} \hat{X}_{t}^{i}+\sum_{i} q_{t-1}^{i} \sigma^{i}+\sum_{i} q_{t-1}^{i} \rho^{i}+\sum_{i} q_{t-1}^{i} \hat{X}_{t}^{i} \rho^{i}+\sum_{i} q_{t-1}^{i} \hat{X}_{t}^{i} \sigma^{i}+\sum_{i} q_{t-1}^{i} \rho^{i} \sigma^{i}+\sum_{i} q_{t-1}^{i} \hat{X}_{t}^{i} \rho^{i} \sigma^{i}
$$

Em que o primeiro termo à direita se refere ao efeito direto, ou também conhecido como efeito do crescimento da produtividade intrassetorial $\left(\sum_{i} q_{t-1}^{i} \hat{X}_{t}^{i}\right)$, o segundo termo se refere ao efeito ocupação, ou também conhecido como mudança estrutural $\left(\sum_{i} q_{t-1}^{i} \sigma^{i}\right)$, o terceiro termo se refere ao efeito preços, que mede a contribuição dos preços relativos para o crescimento da produtividade $\left(\sum_{i} q_{t-1}^{i} \rho^{i}\right)$, e, os últimos quatro termos à direita têm difícil interpretação econômica sendo somente necessários para o processo de manipulação algébrica, desse modo, são somados e chamados de efeito interação. Definidos os procedimentos metodológicos, a seção seguinte apresenta os resultados obtidos.

\section{Resultados e discussões}

Inicialmente, foi feita uma análise tanto para o ano de 2004 quanto para o ano de 2015 em relação aos níveis de produtividade do trabalho, tanto agregado quanto agropecuária, indústria e serviços. Foi feita uma divisão em quatro quartis dos níveis de produtividade gerados pelas unidades federativas em cada setor no ano analisado. Desse modo, foram obtidos os intervalos de alta, alta-média, médiabaixa e baixa produtividade. A intenção foi verificar quais unidades da federação estão incluídas em cada um desses quartis e se esse comportamento continuou tanto no ano de 2004 quanto no ano de 2015.

A Figura 1 apresenta os mapas de quartis analisados. Nos mapas à esquerda estão localizadas as análises para o ano de 2004, e, para comparação, à direita estão localizados os mapas para o ano de 2015. Os mapas superiores dizem respeito à produtividade agregada, sendo seguidos pelos mapas da agropecuária, indústria e serviços.

Ao analisar a produtividade agregada da economia, se verifica que houve algumas alterações na composição das unidades federativas em cada quartil. No 
entanto, de modo geral, é possível detectar certa heterogeneidade regional. Considerando que em parcela significativa do Nordeste há presença de produtividade baixa, em parcela do Centro-Oeste e Sudeste há presença de produtividade média-alta, e em parte do Sul, Sudeste e Centro-Oeste há presença de produtividade alta. Sendo que este comportamento foi verificado como sendo mantido no decorrer do período, características que descrevem heterogeneidade regional conforme mencionado anteriormente. $\mathrm{Na}$ análise do setor da agropecuária, novamente se verifica uma predominância de produtividade baixa no Nordeste, com produtividade média-alta no Norte, e, produtividade alta no Centro-Oeste e Sul, sendo que esse comportamento continuou presente no ano de 2004 a 2015. 
Figura 01 - Mapas de Quartis do Nível da Produtividade para Setores e Unidades

Federativas, 2004 e 2014

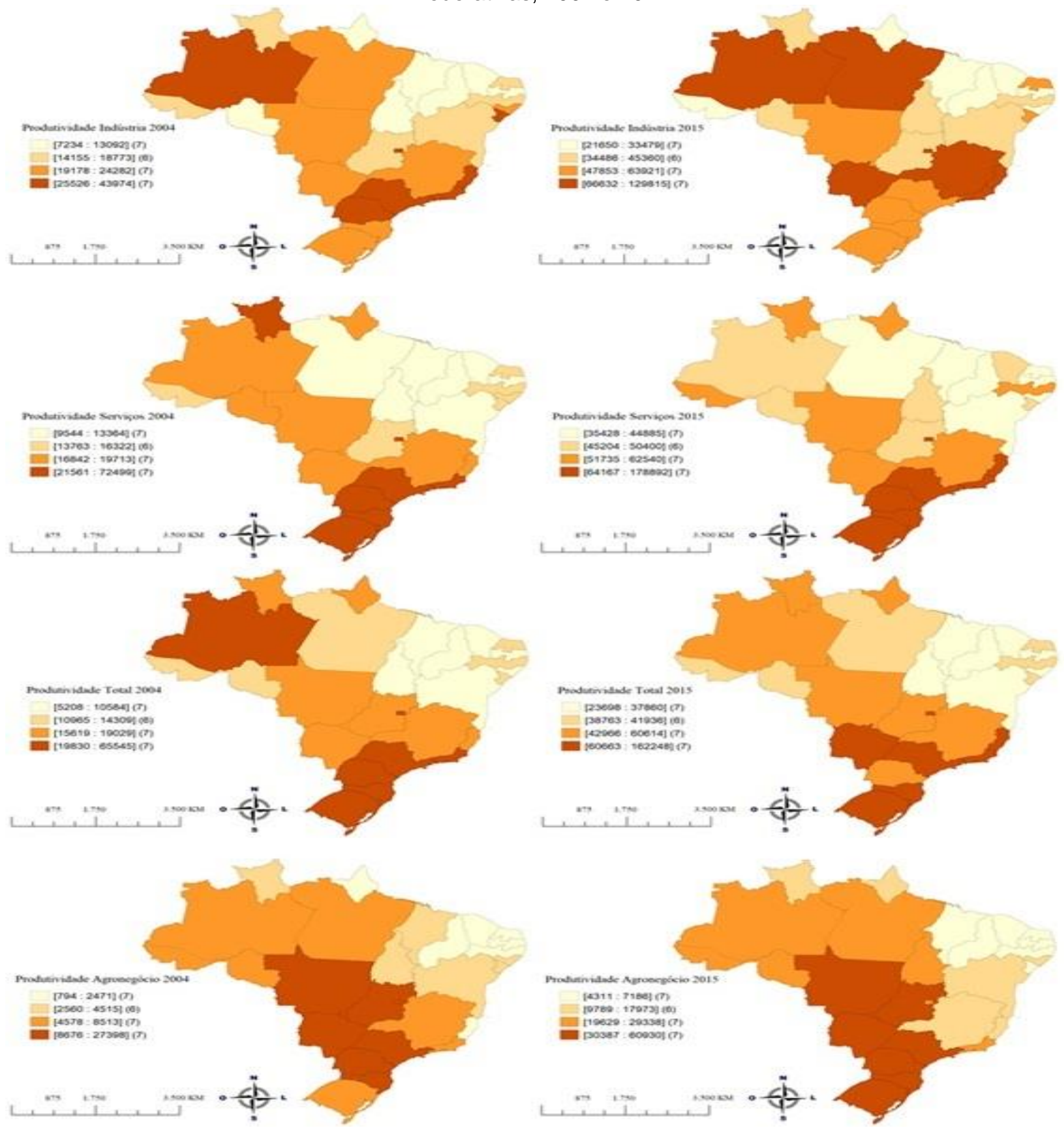

Fonte: elaboração própria utilizando o software Geoda, com base nos dados do IBGE e PNAD (2019).

Ao analisar os mapas do setor industrial, primeiramente se verifica que, em comparação com o setor da agropecuária, há significativamente menos aglomeração de unidades federativas vizinhas com produtividade alta. Ademais, se verifica que, com respeito à produtividade alta, somente houve manutenção do estado do Amazonas neste quartil. Em relação ao quartil de produtividade baixa, se verifica 
que a região Nordeste está parcialmente inclusa neste quartil tanto no ano de 2004 quanto no ano de 2015. A respeito do quartil de produtividade média-alta, se verifica que os estados do Centro-Oeste e Sul estiveram parcialmente presentes em ambos os anos. Por fim, ao analisar o setor de serviços, se verifica a presença de manutenção nos anos de produtividade alta no Sul e Sudeste, produtividade médiaalta no Centro-Oeste e produtividade baixa no Nordeste. Desse modo, são também verificadas características de heterogeneidade regional e estrutural no país.

A Figura 2, por sua vez, apresenta a taxa de crescimento da produtividade no período em cada setor. O primeiro mapa diz respeito ao crescimento agregado, 0 da direita superior se refere ao da agropecuária, o da esquerda inferior representa a indústria e o da direita inferior mostra os dados dos serviços. No primeiro mapa se observa a produtividade agregada, e, ao contrário do que ocorreu com a produtividade em nível, o quartil de valor alto está localizado no Nordeste, e os estados do Sul estão localizados no quartil de valor baixo.

A respeito do setor de agropecuária se verifica que não há aglomeração regional dos estados em cada quartil, sendo a taxa de crescimento da produtividade mais dispersa. O mesmo ocorre com a indústria, porém, mais moderadamente, já que é possível verificar que há presença de estados do Sul e Sudeste nos quartis de produtividade mais baixa, e, presença de estados do Norte e Nordeste nos quartis de produtividade alta. E, por fim, na análise dos serviços, se verifica que houve maior crescimento no Nordeste, com crescimento médio-alto no Norte e CentroOeste, e o Sul e Sudeste tiveram crescimento médio-baixo e baixo, sendo que este setor é o que mais se assemelha ao do crescimento da produtividade agregada, indicando novamente a predominância do setor de serviços no desempenho da produtividade brasileira agregada. 
Figura 02 - Mapas de Quartis do Crescimento da Produtividade por Setor e Unidade Federativa no período de 2004-2015
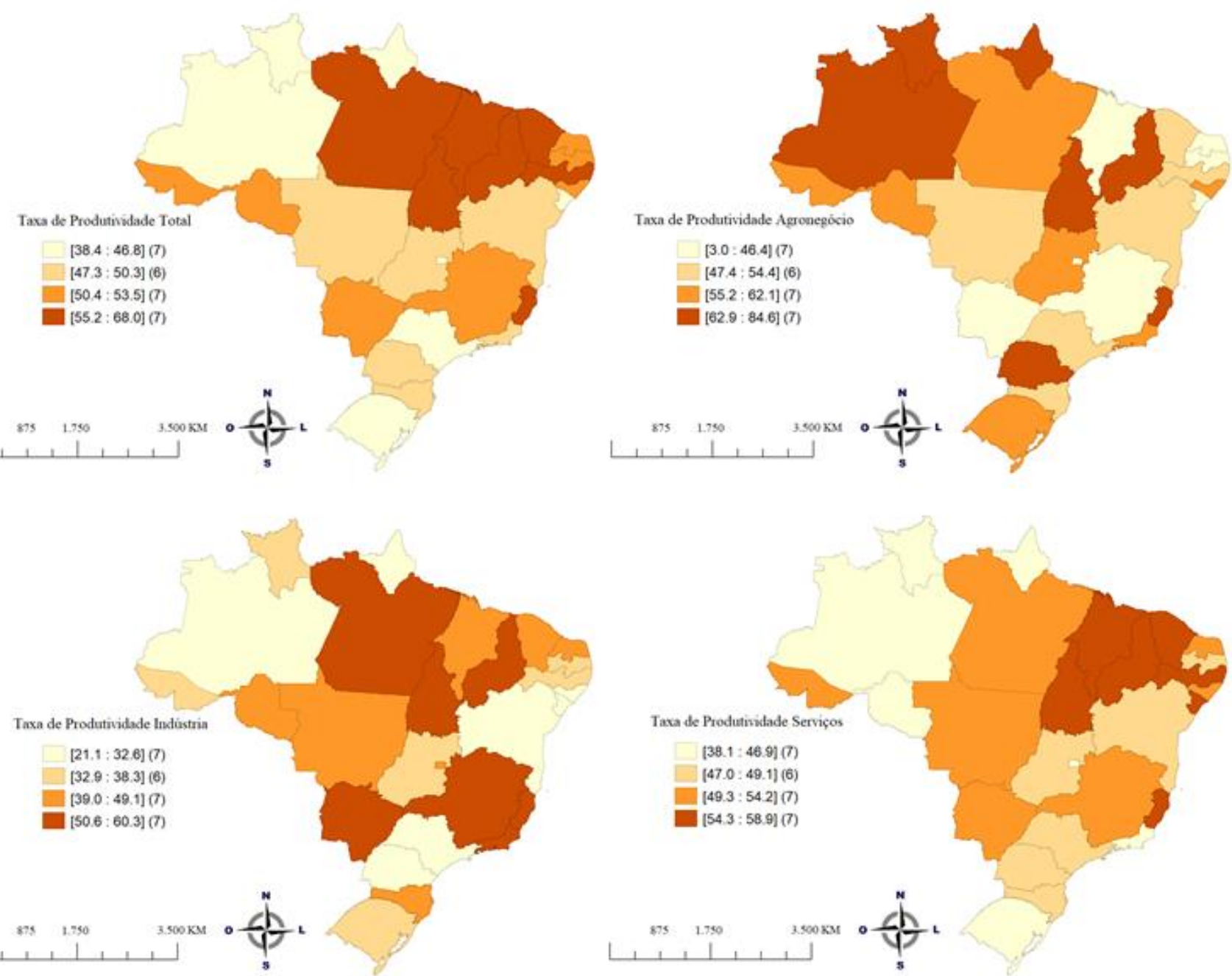

Fonte: elaboração própria utilizando o software Geoda, com base nos dados do IBGE e PNAD (2019).

Portanto, o que se pode verificar da análise dos mapas é que existe heterogeneidade regional, sendo que ao considerar os níveis de produtividade, a região do Nordeste se caracterizou como estando presente no quartil de baixa produtividade e o Sul e Sudeste como estando no quartil de alta produtividade, independente do setor. Já ao analisar o crescimento da produtividade no período o quadro se inverteu, com o Nordeste estando na banda de crescimento mais acelerado e o Sul e Sudeste estando na banda de crescimento mais baixo, independente do setor analisado. Assim, é possível corroborar as conclusões de Squeff e Nogueira (2013) ao afirmarem que nos últimos anos os setores menos dinâmicos são os que estão crescendo mais. No caso da presente análise são as regiões que possuem menor nível de produtividade que estão crescendo mais, ou seja, é possível considerar que no futuro possa existir convergência da produtividade entre as regiões, a questão que se levanta é se esta convergência se daria em 
produtividade alta ou produtividade baixa, conforme questionaria Fochezatto e Stulp (2007).

A Figura 3 apresenta os níveis de produtividade por setor nas grandes regiões tanto no ano de 2004 quanto 2015. Primeiramente, se observa que há diferença nos desempenhos das regiões, independente do setor observado, mais uma vez evidenciando heterogeneidade regional no país.

Figura 03 - Gráficos dos Níveis de Produtividade por Setor e Grande Região, 2004 e 2015
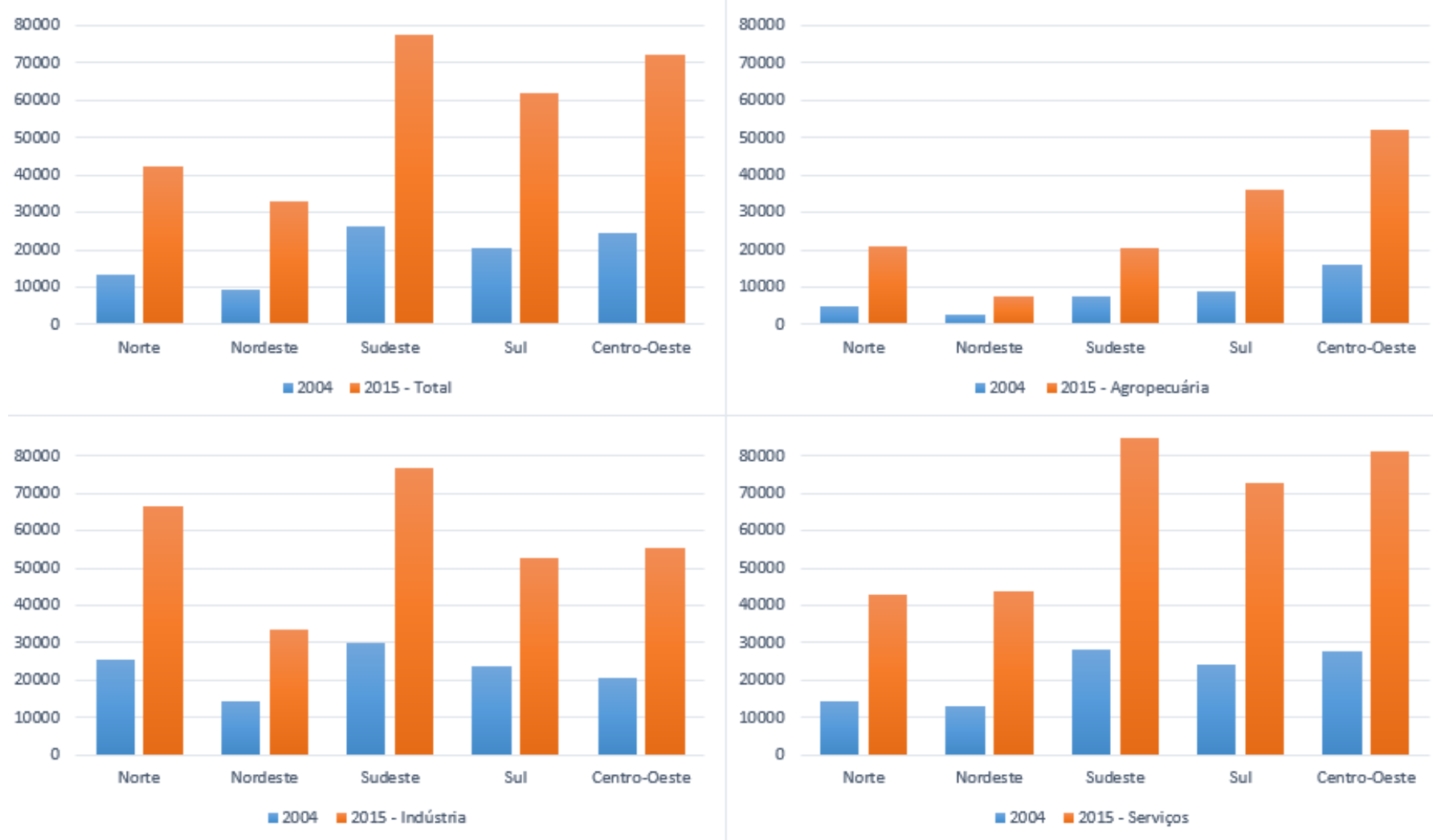

Fonte: elaboração própria com base nos dados do IBGE e PNAD (2019).

A seguir, se verifica que houve crescimento da produtividade em todas as regiões e setores. Porém, mais importante, se verifica que há diferença dos níveis de produtividade entre os setores, sendo que a agropecuária possui menor nível de produtividade, independente da região observada, no entanto, é o setor que mais cresce. Entretanto, este crescimento da produtividade da agropecuária pode ser decorrente do downsizing, conforme afirma Carvalho (2015).

Portanto, ao observar o desempenho dos setores, independentemente da região escolhida para a análise, se verifica que há divergência entre o desempenho setorial da produtividade, e que esta diferença é mantida no tempo, caracterizando a heterogeneidade estrutural no país. Além disso, também se verifica que no ano de 2015 a indústria perdeu espaço para os serviços como o setor mais produtivo, 
lembrando que os serviços são caracterizados por atividades pouco produtivas e mais intensivas em trabalho do que em capital, sendo que este fato também pode ajudar a explicar o pobre desempenho da produtividade do trabalho agregada brasileira,de acordo com Matteo (2015).

A seguir, a Tabela 1 apresenta os resultados obtidos a partir da decomposição do crescimento realizada. Primeiramente se verifica que houve crescimento da produtividade agregada do trabalho no período, independente da unidade federativa observada. Na sequência, se constatou que o efeito direto, ou a produtividade intrassetorial, foi o componente que mais contribuiu para 0 crescimento da produtividade agregada.

Em seguida, o componente que mais contribuiu para o desempenho da produtividade agregada foi o efeito ocupação, ou a mudança estrutural, sendo que contribuiu positivamente (com exceção do Sergipe e do Mato Grosso do Sul, onde a contribuição foi negativa). Por fim, ao verificar a contribuição do efeito preço, o resultado é divergente dependendo do local observado. No geral, se verificou que no Brasil e nas grandes regiões (com exceção do Nordeste), a sua contribuição foi negativa para a produtividade, apesar de também ser uma pequena contribuição.

Portanto, se verificou que o desempenho da produtividade do trabalho agregada no período de 2004 a 2015 foi principalmente sustentado pela contribuição da produtividade intrassetorial, resultado que corrobora os encontrados tanto por Bonelli (2014) quanto por Squeff e De Negri (2014). Sendo que a parcela de contribuição da mudança estrutural foi baixa, lembrando novamente que o desempenho da produtividade brasileira é considerado baixo. Desse modo, o baixo desempenho da produtividade brasileira reside no baixo desempenho da produtividade intrassetorial. Assim, acelerando a mudança estrutural no país, ou seja, a maior migração da mão de obra de setores pouco dinâmicos para setores mais dinâmicos, pode contribuir positivamente para o crescimento da produtividade e elevar o seu desempenho a níveis satisfatórios.

Tabela 01 - Decomposição do Crescimento da Produtividade do Trabalho, 2004-2015

\begin{tabular}{l|cc|cc|cc|cc|cc}
\hline Local & \multicolumn{2}{|c|}{ Produtividade } & \multicolumn{2}{|c|}{ Direto } & \multicolumn{2}{|c|}{ Ocupação } & \multicolumn{2}{|c|}{ Preços } & \multicolumn{2}{|c}{ Interação } \\
\hline N & 2,17 & 100 & 2,00 & $(92,19)$ & 0,07 & $(3,05)$ & $-0,0006$ & $(-0,03)$ & 0,10 & $(4,78)$ \\
\hline RO & 2,19 & 100 & 1,94 & $(88,30)$ & 0,12 & $(5,35)$ & 0,0023 & $(0,10)$ & 0,14 & $(6,24)$ \\
AC & 2,35 & 100 & 2,19 & $(93,32)$ & 0,06 & $(2,76)$ & $-0,0017$ & $(-0,07)$ & 0,09 & $(3,99)$ \\
AM & 1,42 & 100 & 1,37 & $(96,37)$ & 0,03 & $(1,83)$ & 0,0041 & $(0,29)$ & 0,02 & $(1,51)$ \\
RR & 1,76 & 100 & 1,59 & $(90,27)$ & 0,11 & $(6,38)$ & 0,0026 & $(0,15)$ & 0,06 & $(3,20)$ \\
PA & 2,61 & 100 & 2,45 & $(93,59)$ & 0,06 & $(2,28)$ & 0,0023 & $(0,09)$ & 0,11 & $(4,04)$ \\
AP & 1,69 & 100 & 1,67 & $(98,73)$ & 0,01 & $(0,79)$ & 0,0103 & $(0,61)$ & 0,00 & $(-0,13)$ \\
TO & 2,99 & 100 & 2,77 & $(92,81)$ & 0,07 & $(2,19)$ & $-0,0060$ & $(-0,20)$ & 0,16 & $(5,19)$ \\
\hline NE & 2,51 & 100 & 2,11 & $(83,99)$ & 0,14 & $(5,38)$ & 0,0044 & $(0,18)$ & 0,26 & $(10,45)$ \\
\hline MA & 2,57 & 100 & 2,47 & $(96,26)$ & 0,04 & $(1,58)$ & 0,0043 & $(0,17)$ & 0,05 & $(2,00)$
\end{tabular}




\begin{tabular}{l|rr|rc|cc|cc|cc} 
PI & 3,79 & 100 & 2,95 & $(77,93)$ & 0,24 & $(6,37)$ & $-0,0006$ & $(-0,02)$ & 0,59 & $(15,72)$ \\
CE & 2,97 & 100 & 2,48 & $(83,54)$ & 0,13 & $(4,48)$ & 0,0039 & $(0,13)$ & 0,35 & $(11,84)$ \\
RN & 2,43 & 100 & 2,06 & $(84,97)$ & 0,11 & $(4,64)$ & $-0,0010$ & $(-0,04)$ & 0,25 & $(10,43)$ \\
PB & 2,28 & 100 & 1,87 & $(82,15)$ & 0,14 & $(6,29)$ & 0,0032 & $(0,14)$ & 0,26 & $(11,42)$ \\
PE & 2,82 & 100 & 2,41 & $(85,24)$ & 0,15 & $(5,43)$ & 0,0090 & $(0,32)$ & 0,25 & $(9,01)$ \\
AL & 2,35 & 100 & 1,89 & $(80,44)$ & 0,23 & $(9,74)$ & 0,0053 & $(0,23)$ & 0,23 & $(9,59)$ \\
SE & 1,71 & 100 & 1,80 & $(105,29)$ & $-0,01$ & $(-0,67)$ & $-0,0007$ & $(-0,04)$ & $-0,08$ & $(-4,58)$ \\
BA & 2,17 & 100 & 1,77 & $(81,61)$ & 0,16 & $(7,23)$ & 0,0035 & $(0,16)$ & 0,24 & $(11,00)$ \\
\hline SE & 1,92 & 100 & 1,85 & $(96,37)$ & 0,02 & $(0,96)$ & $-0,0057$ & $(-0,30)$ & 0,06 & $(2,97)$ \\
\hline MG & 2,22 & 100 & 2,16 & $(97,36)$ & 0,01 & $(0,66)$ & $-0,0033$ & $(-0,15)$ & 0,05 & $(2,14)$ \\
ES & 3,05 & 100 & 2,74 & $(90,04)$ & 0,09 & $(2,80)$ & 0,0197 & $(0,65)$ & 0,20 & $(6,51)$ \\
RJ & 2,07 & 100 & 2,06 & $(99,70)$ & 0,01 & $(0,26)$ & 0,0055 & $(0,26)$ & 0,00 & $(-0,22)$ \\
SP & 1,74 & 100 & 1,67 & $(96,07)$ & 0,02 & $(1,02)$ & 0,0018 & $(0,10)$ & 0,05 & $(2,81)$ \\
\hline S & 2,01 & 100 & 1,89 & $(94,02)$ & 0,06 & $(3,11)$ & $-0,0010$ & $(-0,05)$ & 0,06 & $(2,92)$ \\
\hline PR & 1,97 & 100 & 1,93 & $(97,61)$ & 0,06 & $(3,24)$ & 0,0015 & $(0,08)$ & $-0,02$ & $(-0,92)$ \\
SC & 2,16 & 100 & 1,99 & $(91,83)$ & 0,07 & $(3,14)$ & $-0,0049$ & $(-0,23)$ & 0,11 & $(5,26)$ \\
RS & 1,94 & 100 & 1,78 & $(92,12)$ & 0,07 & $(3,43)$ & 0,0028 & $(0,15)$ & 0,08 & $(4,30)$ \\
\hline CO & 1,97 & 100 & 1,94 & $(98,31)$ & 0,02 & $(0,80)$ & $-0,0021$ & $(-0,11)$ & 0,02 & $(1,00)$ \\
\hline MS & 2,34 & 100 & 2,35 & $(100,67)$ & $-0,01$ & $(-0,27)$ & $-0,0038$ & $(-0,16)$ & $-0,01$ & $(-0,23)$ \\
MT & 2,19 & 100 & 2,20 & $(100,78)$ & 0,00 & $(-0,13)$ & $-0,0120$ & $(-0,55)$ & 0,00 & $(-0,11)$ \\
GO & 1,97 & 100 & 1,96 & $(99,56)$ & 0,01 & $(0,71)$ & 0,0011 & $(0,06)$ & $-0,01$ & $(-0,32)$ \\
DF & 1,48 & 100 & 1,46 & $(99,07)$ & 0,01 & $(0,47)$ & $-0,0015$ & $(-0,10)$ & 0,01 & $(0,56)$ \\
\hline BR & 2,07 & 100 & 1,89 & $(91,57)$ & 0,06 & $(2,96)$ & $-0,0031$ & $(-0,15)$ & 0,12 & $(5,61)$ \\
\hline FOn
\end{tabular}

Fonte: elaboração própria com base nos dados do IBGE e PNAD (2019).

Ademais, também se verificou que relacionado ao crescimento da produtividade no período, o maior crescimento foi encontrado nas regiões Norte e Nordeste. Novamente, estes resultados corroboram os encontrados por Matteo (2015) e Nogueira, Infante e Mussi (2014), ao dissertarem que as regiões que são caracterizadas por menor nível de produtividade (Norte e Nordeste), são as que estão crescimento mais rapidamente. Isso pode sugerir a existência de um processo de convergência de produtividade no país, conforme defendido por Fochezatto e Stulp (2007). Por fim, ao se verificar que as unidades federativas com maior nível de produtividade estão obtendo crescimento comparativo menor no período, e que ao decompô-las o item que mais as representa é o de produtividade intrassetorial, é possível concluir que maiores mudanças na estrutura produtiva poderiam intensificar o crescimento da eficiência produtiva nestas unidades federativas, de acordo com o destacado por Bonelli (2014).

Concluindo, os resultados demonstram que ao se realizar uma análise da produtividade do trabalho brasileira por meio das unidades federativas e macrossetores, foi possível confirmar a hipótese de existência de heterogeneidade estrutural e heterogeneidade regional. Além de destacar que a maior contribuição do crescimento da eficiência produtiva parte da produtividade intrassetorial. De modo que estímulos em termos de mudança na estrutura produtiva das unidades 
federativas seriam benéficos para a melhora do desempenho da produtividade das respectivas unidades e também no agregado.

\section{Considerações Finais}

O objetivo do presente trabalho foi analisar os efeitos de mudanças na estrutura produtiva sobre a produtividade agregada, além de destacar se há existência de heterogeneidade estrutural e regional entre as unidades federativas, considerando que há certo consenso na literatura de que o desempenho da produtividade brasileira não é considerado satisfatório.

Com base na breve revisão de literatura realizada, foi possível constatar a importância da produtividade do trabalho para o crescimento e desenvolvimento econômico dos países, e, que o desempenho da produtividade brasileira é considerado baixo por ser sustentado nos últimos anos por setores menos dinâmicos, sendo que o setor que mais cresceu foi a agropecuária, respectivamente o setor que possui menor nível de produtividade. Além disso, os autores também entraram em certo consenso de que existe heterogeneidade estrutural e regional no país.

No presente artigo, ao realizar uma análise para um período mais recente na economia e de forma mais desagregada possível (unidades federativas), buscou-se estudar o desempenho da produtividade brasileira por setores. O que se constatou é que existe heterogeneidade regional no país, sendo que a região do Nordeste é mais caracterizada por desempenho baixo no nível de produtividade, e o eixo de estados do Centro-Oeste, Sudeste e Sul é caracterizado por desempenho alto, independentemente do setor analisado. Ademais, ao se verificar o crescimento do período, se verificou que o Nordeste possuiu o crescimento mais alto do país, e no Sul e Sudeste o crescimento foi mais baixo, novamente independente do setor analisado. Assim, se verificou que as regiões com o desempenho mais baixo estão crescendo mais e as regiões com desempenho mais alto estão crescendo pouco, o que pode sugerir que no futuro poderia ocorrer um processo de convergência de produtividade.

Ao analisar o desempenho dos setores nas grandes regiões também se verificou que há divergência na produtividade dos setores, sendo que a agropecuária possui menor nível de produtividade e é o setor que mais tem crescido no período, e a indústria deixou de ser o setor mais produtivo para os serviços e tem tido baixo crescimento no período. Portanto, é possível concluir que o baixo desempenho da 
produtividade agregada brasileira tem sido causado pelo maior crescimento dos setores menos dinâmicos em detrimento de setores mais dinâmicos. Assim, é possível concluir que existe heterogeneidade estrutural, além da heterogeneidade regional no Brasil.

Ademais, ao se verificar como se decompôs o crescimento da produtividade agregada no período, se verificou que apesar de haver crescimento positivo em todas as unidades federativas, o seu desempenho foi baixo e que foi principalmente causado pela produtividade intrassetorial, ou seja, por haver pouco crescimento da produtividade dentro dos setores, o crescimento agregado foi baixo. Além disso, se verificou que a contribuição da mudança estrutural (efeito-ocupação) foi positivo (com duas exceções), no entanto, esta contribuição foi mínima para o desempenho da produtividade agregada. Ou seja, se houver aceleração do processo de mudança estrutural (migração de mão de obra de setores menos dinâmicos para setores mais dinâmicos), é possível que haja reflexos positivos no desempenho da produtividade agregada. Por fim, a contribuição do efeito preços foi divergente entre as unidades federativas, sendo que no Brasil como um agregado o seu desempenho foi negativo, porém ínfimo.

Concluindo, o país é caracterizado por heterogeneidade estrutural e regional rígida e baixo desempenho da produtividade do trabalho. Contudo, se verificou que a contribuição da mudança estrutural para a produtividade agregada foi pequeno. Assim, através da aceleração do efeito ocupação, é possível que haja melhoras no desempenho da produtividade agregada brasileira, o que poderia levar a melhoras em termos de crescimento econômico de longo prazo.

\section{REFERÊNCIAS}

BAUMOL, W. J. (1967). Macroeconomics of Unbalanced Growth: the anatomy of urban crisis. The American Economic Review, p. 415-426.

BONELLI, R. (2014). Produtividade e Armadilha do Lento Crescimento. In: DE NEGRI, F.; CAVALCANTE, L. R. (Org.). Produtividade no Brasil: desempenho e determinantes, v. 1, 1 ed. Brasília: IPEA:ABDI, p. 111-142.

BONELLI, R.; BACHA, E. (2013). Crescimento Brasileiro Revisitado. In: VELOSO, F. A. et al. Desenvolvimento Econômico: uma perspectiva brasileira. Rio de Janeiro: Ed. Campus.

BONELLI, R.; VELOSO, F. (2012). Rio de Janeiro: crescimento econômico e mudança estrutural: In: PINHEIRO, A. C.; VELOSO, F. Rio de Janeiro: um estado em transição. Rio de Janeiro: Editora FGV. 
CAVALCANTE, L. R.; DE NEGRI, F. (2014). Evolução Recente dos Indicadores de Produtividade no Brasil. In: DE NEGRI, F.; CAVALCANTE, L. R. (Org.). Produtividade no Brasil: desempenho e determinantes, v. 1, 1 ed. Brasília: IPEA:ABDI, p. 143-171.

DE NEGRI, F.; CAVALCANTE, R. (2014). Os Dilemas e os Desafios da Produtividade no Brasil. In: DE NEGRI, F.; CAVALCANTE, R.(Org). Produtividade no Brasil: desempenho e determinantes, v.1, 1 ed. Brasília: IPEA:ABDI, p. 15-52.

FOCHEZATTO, A; STULP, V. J. (2007). Análise da Dinâmica da Produtividade do Trabalho entre Setores e Estados Brasileiros na Década de 90. Análise Econômica, v. 25, n. 48, p. 99-122.

GALEANO, E. A. V.; WANDERLEY, L. A. (2013). Produtividade Industrial do Trabalho e Intensidade Tecnológica nas Regiões do Brasil: uma análise regional e setorial para 0 período 1996-2007. Planejamento e Políticas Públicas, n. 40, jan./jun.

INSTITUTO BRASILEIRO DE GEOGRAFIA E ESTATÍSTICA (IBGE). (2019). Dados. Disponível em: <www.ibge.gov.br/>. Acesso em 31 jan. 2019.

KALDOR, N. (1978). Causes of the Slow Rate of Economic Growth in the United Kingdom. In: KALDOR, Nicholas. Further Essays on Economic Theory, Londres: The Anchor Press Ltd.

MATTEO, M. (2015). Heterogeneidade Regional. In: INFANTE, R.; MUSSI, C.; ODDO, M. (Org.). Por um Desenvolvimento Inclusivo: o caso do Brasil. Santiago: CEPAL, p. 235254.

NOGUEIRA, M. O.; INFANTE, R.; MUSSI, C. (2014). Produtividade do Trabalho e Heterogeneidade Estrutural no Brasil Contemporâneo. In: DE NEGRI, F.; CAVALCANTE, R.(Org). Produtividade no Brasil: desempenho e determinantes, v.1, 1 ed. Brasília: IPEA:ABDI, p. 15-52.

PESQUISA NACIONAL POR AMOSTRA DE DOMICÍLIOS (PNAD). (2019). Microdados de 2004 a 2015. Disponível em:

<ftp://ftp.ibge.gov.br/Trabalho_e_Rendimento/Pesquisa_Nacional_por_Amostra_de_Domicili os_anual/>. Acesso em 24 jan. 2019.

SQUEFF, G. C.; DE NEGRI, F. (2014). Produtividade do Trabalho e Mudança Estrutural no Brasil nos Anos 2000. In: DE NEGRI, F.; CAVALCANTE, L. R. (Org.). Produtividade no Brasil: desempenho e determinantes, v. 1, 1 ed. Brasília: IPEA:ABDI, p. 249-280.

SQUEFF, G. C.; NOGUEIRA, M. O. (2013). A Heterogeneidade Estrutural no Brasil de 1950 a 2009. Textos para Discussão Cepal - Ipea 51. Brasília: Cepal-Ipea.

THIRLWALL, A. P. (1983). A Plain Man's Guide to Kaldor's Growth Laws. Journal of Post Keynesian Economics, v. 5, n. 3, p. 345-358.

VELOSO, F.; MATOS, S.; COELHO, B. (2015). Produtividade do Trabalho no Brasil: uma análise setorial. Texto de Discussão n. 85, FGV: IBRE. 


\section{NOTAS DE AUTOR}

\section{CONTRIBUIÇÃO DE AUTORIA}

Thais Andreia Araujo de Souza - Concepção. Coleta de dados, Análise de dados, Elaboração do manuscrito. Marieli Vieira - Elaboração do manuscrito, Participação ativa da discussão dos resultados e revisão. Marina Silva da Cunha - Elaboração do manuscrito, revisão e aprovação da versão final do trabalho.

\section{FINANCIAMENTO}

Não se aplica.

\section{CONSENTIMENTO DE USO DE IMAGEM}

Não se aplica.

\section{APROVAÇÃO DE COMITÊ DE ÉTICA EM PESQUISA}

Não se aplica.

\section{CONFLITO DE INTERESSES}

Nenhum.

\section{LICENÇA DE USO}

Este artigo está licenciado sob a Licença Creative Commons CC-BY. Com essa licença você pode compartilhar, adaptar, criar para qualquer fim, desde que atribua a autoria da obra.

\section{HISTÓRICO}

Recebido em: 25-10-2019

Aprovado em: 05-05-2020 